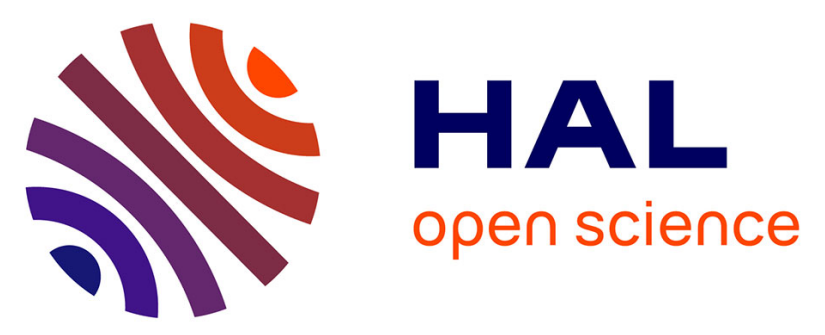

\title{
Asymmetric Dressing of WSe2 with (Macro)molecular Switches: Fabrication of Quaternary-Responsive Transistors
}

Haixin Qiu, Stefano Ippolito, Agostino Galanti, Zhaoyang Liu, Paolo Samorì

\section{- To cite this version:}

Haixin Qiu, Stefano Ippolito, Agostino Galanti, Zhaoyang Liu, Paolo Samorì. Asymmetric Dressing of WSe2 with (Macro)molecular Switches: Fabrication of Quaternary-Responsive Transistors. ACS Nano, 2021, 15 (6), pp.10668-10677. 10.1021/acsnano.1c03549 . hal-03270555

\section{HAL Id: hal-03270555 \\ https://hal.science/hal-03270555}

Submitted on 24 Jun 2021

HAL is a multi-disciplinary open access archive for the deposit and dissemination of scientific research documents, whether they are published or not. The documents may come from teaching and research institutions in France or abroad, or from public or private research centers.
L'archive ouverte pluridisciplinaire HAL, est destinée au dépôt et à la diffusion de documents scientifiques de niveau recherche, publiés ou non, émanant des établissements d'enseignement et de recherche français ou étrangers, des laboratoires publics ou privés. 


\section{Asymmetric Dressing of $\mathrm{WSe}_{2}$ with}

\section{(macro)molecular switches: fabrication of}

\section{Quaternary-Responsive Transistors}

Haixin Qiu, Stefano Ippolito, Agostino Galanti, Zhaoyang Liu, Paolo Samorì

Université de Strasbourg, CNRS, ISIS, 8 alleé Gaspard Monge, 67000 Strasbourg, France

\section{ABSTRACT}

The forthcoming saturation of Moore's law has led to a strong demand for integrating analog functionalities within semiconductor-based devices. As a step towards this goal, we fabricate quaternary-responsive $\mathrm{WSe}_{2}$-based field-effect transistors (FETs) whose output current can be remotely and reversibly controlled by light, heat and electric field. A photochromic silaneterminated spiropyran (SP) is chemisorbed on $\mathrm{SiO}_{2}$ forming a self-assembled monolayer (SAM) that can switch from the SP to the merocyanine (MC) form in response to UV illumination and switch back by either heat or visible illumination. Such SAM is incorporated at the dielectric-semiconductor interface in $\mathrm{WSe}_{2}$-based FETs. Upon UV irradiation, a drastic decrease in the output current of $82 \%$ is observed and ascribed to the zwitterionic MC isomer acting as charge scattering site. To impart an additional functionality, the $\mathrm{WSe}_{2}$ top surface is coated with a ferroelectric co-polymer layer based on poly(vinylidene fluoride-cotrifluoroethylene) (P(VDF-TrFE)). Due to its switchable inherent electrical polarization, it can promote either the accumulation or depletion of charge carriers in the $\mathrm{WSe}_{2}$ channel, thereby 
inducing a current modulation with $99 \%$ efficiency. Thanks to the efficient tuning sparked by the two components and their synergistic effects, the device polarity could be modulated from n-type to p-type. Such a control over the carrier concentration and device polarity is key to develop 2D advanced electronics. Moreover, the integration strategy of multiple stimuliresponsive elements into a single FET allows to greatly enrich its functionality, thereby promoting the development for More-than-Moore technology.

KEYWORDS: 2D semiconductors, spiropyran, multi-stimuli-responsive, photochromic, thermochromic, ferroelectric 
Taking advantage of their extraordinary physicochemical properties and compatibility with silicon-based technology, two-dimensional materials (2DMs), including graphene and transition metal dichalcogenides, have been the target of extensive fundamental studies and technological progress over the past decade. ${ }^{1-3}$ In the current More-than-Moore era, the construction of multifunctional and multiresponsive 2DM-based devices is being explored. Owing to their atomic thickness and reduced screening effect, the intrinsic properties of 2DMs are extremely sensitive to the external environment and can be modulated by surface modification. ${ }^{4}$ Various organic molecules have already been embedded into 2DM-based electronic devices (e.g. transistors) as a mean to controllably adjust their electrical characteristics, mainly via doping. ${ }^{5-7}$ In addition, during the last five years considerable efforts have been dedicated to the incorporation of stimuli-responsive molecules, which are able to undergo precise nanoscale rearrangements in response to chemical or physical inputs, as fundamental building blocks in conventional electronic devices. ${ }^{8}$ Upon integration of such dynamic molecular systems, the external stimuli may act as an additional remote control to operate the target devices, endowing them with ad hoc properties for specific applications such as memories, ${ }^{9,10} \mathrm{UV}$ sensors,${ }^{11,12}$ synapse simulation, ${ }^{13,14}$ and so on.

Light and electric field recently emerged as attractive external stimuli, both of which are noninvasive, remotely and digitally controllable with high spatial and temporal resolution. ${ }^{15}$ As a mild and easy-to-use stimulus, temperature also represents one of the most widely explored inputs in the design of stimuli-responsive platforms.

Triggered by light, the isomerization of photochromic molecules has been demonstrated to be a simple-but-effective strategy to implement the optical switching into the manipulation of charge carrier transport in 2DM-based FETs. ${ }^{16} \mathrm{We}$ recently reported the successful fabrication of optically switchable FETs by the physisorption of a top layer of either diarylethenes, ${ }^{17,}{ }^{18}$ 
azobenzene ${ }^{19-22}$ or spiropyran (SP) $)^{23-26}$ onto the $2 \mathrm{D}$ channel material, demonstrating a reversible control of their output current as a result of the molecular photoisomerization. On the other hand, an external electric field was used to control the intrinsic bistable polarization of ferroelectric macromolecules, such as the ferroelectric co-polymer poly(vinylidene fluorideco-trifluoroethylene), $\mathrm{P}(\mathrm{VDF}-\mathrm{TrFE})$, when interfaced to $2 \mathrm{DMs}$, as a mean to reversibly modulate the doping leve ${ }^{27,28}$ according to the direction of its polarization and device current output..$^{29-31}$

In this paper, we upgrade such stimuli-responsive 2DM-based FETs to a higher level by "dressing" the two surfaces of the ultrathin channel materials in an asymmetric manner, yielding a Janus structure. This enables the incorporation of a third component into the device geometry, where the bottom and the top surface of the 2DM interact with different functional molecules. The presence of more than one stimuli-responsive function in the FET imparts to the device an overall multi-stimuli-responsive character. To enhance the robustness of the photochromic layer and render it compatible with standard photolithography processes for optoelectronic devices fabrication, the chemisorption of a SP derivative bearing an organosilane moiety capable to bind the $\mathrm{Si} / \mathrm{SiO}_{2}$ substrate and form a self-assembled monolayer (SAM) is utilized to incorporate such a functional molecule at the dielectric-semiconductor interface.$^{32}$ This chemisorption approach facilitates the device fabrication and provides a better compatibility when integrating a third responsive element into the system.

Here, we sandwich a few-layer thick $\mathrm{WSe}_{2}$ flake in between a SAM of SP molecules chemisorbed on $\mathrm{Si} / \mathrm{SiO}_{2}$ substrate and a top coating layer of the ferroelectric co-polymer $\mathrm{P}(\mathrm{VDF}-\mathrm{TrFE})$. The photoisomerization reaction from SP to MC can be triggered by UV irradiation, while the reverse isomerization from MC to SP can be accomplished by visible (vis) irradiation as well as heating, ${ }^{33}$ adding a further thermal-responsive nature to the related FET 
devices. Therefore, such a vertically asymmetric architecture enables an independent control of the output current under the effect of distinct multiple inputs, yielding a quaternary-stimuliresponsive FETs capable of responding to heat, light and electric field. The electron current modulation efficiency reaches $84 \%$ for SP photoisomerization, and $99 \%$ for $\mathrm{P}(\mathrm{VDF}-\mathrm{TrFE})$ polarization. Moreover, taking advantage of the synergistic effect between the two stimuli, the electrons in the channel can be totally depleted when combining them into a single switch and the device polarity can be modulated from n-type to p-type. Furthermore, by collecting a pool of multiple stimuli as inputs, the overreliance upon a single stimulus has been reduced, increasing the sensitivity and compliance of the molecular-tailored stimuli-responsive platform.

\section{Results and Discussion}

The synthesis of the silane-terminated SP derivative and the protocol of SP SAM functionalization of $\mathrm{SiO}_{2}$ is provided in Experimental Methods and in the Supporting Information (Scheme S1, Figure S1-S5). The reversible photoisomerization between SP and MC is schematically illustrated in Figure 1a. The successful functionalization of SP SAM on $\mathrm{SiO}_{2}$ surface was confirmed through UV-vis absorption spectra (Figure 1, Figure S6-S7), water contact angle measurements (Figure S8), atomic force microscopy (AFM) (Figure S9) and photoluminescence (PL) mapping (Figure 2 and Figure S10-S11).

UV-vis absorption spectra of SP SAM on quartz substrate were initially recorded during the course of UV irradiation (Figure 2a). The typical band of MC chromophores centered at 550 nm appeared after a few seconds of UV irradiation. Therefore, the kinetics of the photoisomerization between SP and MC could be followed by monitoring the evolution of the absorbance at $550 \mathrm{~nm}\left(\mathrm{Abs}_{550 \mathrm{~nm}}\right)$. Abs $\mathrm{Ab}_{50 \mathrm{~nm}}$ kept rising while irradiating with UV light until reaching the photostationary state (PSS) after $180 \mathrm{~s}$, the trend of which can be fitted with a simple monoexponential equation (Figure $2 \mathrm{~b}$ and Table S1). ${ }^{24}$ Note that the saturation time of 
SP to MC transition is dependent on the intensity of UV illumination, as shown in Figure S7. Being photochromic and thermochromic, the MC isomer can be switched back to SP by either thermal treatment or vis irradiation, showing a decrease of $\mathrm{Abs}_{550 \mathrm{~nm}}$ in accordance to a firstorder decay (Figure S6). At room temperature, $c a .37 .5 \%$ of the molecules in the MC form were transformed to SP after 11 hours (Figure 2c). This process can be accelerated by heating at $c a .80^{\circ} \mathrm{C}$, with a kinetic that requires only $4 \min$ (Figure $2 \mathrm{~d}$ ) for the complete conversion of MC to SP. Irradiation with vis light for $c a .45$ min enabled to convert all the MC into SP (Figure 2e). Finally, when the 3 treatments were combined together within the same experiment, different kinetics could be clearly singled out (Figure 2f). The different kinetics can be explained with the fact that the thermal and photochemical isomerization of MC to SP follow different reaction mechanisms..$^{34,35}$

Water contact angle measurements of unmodified $\mathrm{Si} / \mathrm{SiO}_{2}$, pristine SP SAMs, and UV/heattreated SP SAMs on $\mathrm{Si} / \mathrm{SiO}_{2}$ surfaces are displayed in Figures $\mathrm{S} 8$. The unmodified $\mathrm{Si} / \mathrm{SiO}_{2}$ surface displayed a hydrophilic behavior $\left(33.6 \pm 0.3^{\circ}\right)$. Upon SP functionalization the hydrophobicity of the surface increased $\left(73.8 \pm 0.6^{\circ}\right)$. When irradiated with UV light, the contact angle of such a surface showed a decrease by $15.2^{\circ}$, indicating a higher hydrophilicity of the surface. This can be explained by the higher polarity of the zwitterionic MC compared to $\mathrm{SP},{ }^{33}$ thus leading to an increase in the wettability of the surface. The initial values could be recovered by following heating, confirming the reversible nature of the SP - MC isomerization.

The morphology of the SP SAM was investigated by AFM (Figure S9). The imaging performed on SP-functionalized $\mathrm{SiO}_{2}$ surface revealed a rather flat morphology, with a root-mean-square roughness $\left(\mathrm{R}_{\mathrm{RMS}}\right)$, determined over an area of $1 \mu \mathrm{m} \times 1 \mu \mathrm{m}$, corresponding to $c a .0 .45 \mathrm{~nm}$. For the sake of comparison, the morphology on unmodified $\mathrm{Si} / \mathrm{SiO}_{2}$ surface was found being smoother with a $\mathrm{R}_{\mathrm{RMS}}$ amounting to $c a .0 .16 \mathrm{~nm}$. As estimated by topographical profile, the 
height of the features displayed by the SP-functionalized $\mathrm{SiO}_{2}$ surface was $1.3 \pm 0.5 \mathrm{~nm}$. We ascribe the presence of such features to aggregates of SP on top of the SP SAM. The appearance of such aggregates is likely due to polymerization of the silane-terminated SP that could not be washed away after the treatment. ${ }^{36}$

The $\mathrm{WSe}_{2}$ flakes were transferred on top of the SP-functionalized $\mathrm{SiO}_{2}$ substrate by using the conventional scotch-tape approach. To unveil the interaction between $\mathrm{SP}$ and $\mathrm{WSe}_{2}, 2 \mathrm{D}$ mapping of photoluminescence (PL) intensity was carried out over $\mathrm{WSe}_{2}$ flakes with different thickness by using an optical microscope equipped with a spectrometer and a $532 \mathrm{~nm}$ laser line. The PL evolution of the SP/MC molecules was first investigated by exploring the wavelength range from $560 \mathrm{~nm}$ to $650 \mathrm{~nm}$ in which $\mathrm{MC}$ is luminescent, while SP is not. As illustrated in Figure $3 \mathrm{a}$, the as-prepared $\mathrm{SP} / \mathrm{WSe}_{2}$ was found to be scarcely photoluminescent. After UV illumination, the PL intensity in the regions that were not covered by the flake strongly increased, corroborating an effective SP to MC isomerization. Interestingly, the region covered by the flake showed a weaker PL, which can be ascribed to the quenching effect by $\mathrm{WSe}_{2}$. Note that a relatively low laser power $(0.03 \mathrm{~mW})$ was utilized during the measurement in order to minimize the occurrence of the $\mathrm{MC} \rightarrow$ SP photoisomerization. In order to switch the molecules back to SP form, a higher laser power $(4.92 \mathrm{~mW})$ was utilized to scan the same region. Figure 3a clearly shows that the PL after laser scanning turned back to the original state, providing evidence for the recovery of SP. Figure 3b depicts the detailed PL spectra evolution in the points labeled as A and B. Note that some spikes emerged around $550 \mathrm{~nm}$, which can be attributed to the Raman signal of both silicon substrate and SP/MC. ${ }^{37}$ To investigate if the SP photoswitch leads to a modulation in the optical properties of the 2DM, we also measured the $\mathrm{PL}$ of $\mathrm{WSe}_{2}$ in the wavelength range from $750 \mathrm{~nm}$ to $900 \mathrm{~nm}$. The PL spectra recorded on a bilayer (highlighted in C) and a few-layer (highlighted in D) region of a $\mathrm{WSe}_{2}$ flake are shown in Figure $3 \mathrm{c}$ and $3 \mathrm{~d}$ (some spatial variations in the PL intensity were observed due to the 
different flake thickness). The bilayer region presents PL spectra with a broad band corresponding to emission from both the indirect and direct bandgaps.$^{38}$ Conversely, the fewlayer region exhibits a strikingly decreased emission intensity compared to the thinner region, and the PL spectra contain two distinct peaks due to the indirect and direct bandgaps emission, as well..$^{38}$ It is worth mentioning that these two regions provided identical results: an overall quenching of the PL intensity for $\mathrm{WSe}_{2}$ was observed when molecules were switched to the MC form, which can then be completely restored by laser irradiation to switch back the molecules to SP form, providing evidence of the electronic interaction between the two components. Importantly, when then same experimental procedure was repeated on flakes with different thickness, it returned the same PL intensity evolution behavior, thus confirming the reproducibility of such an evolution (Figure S10-S11). We ascribe the variation of the PL properties of $\mathrm{WSe}_{2}$ to the change in dipole moment of the underlying $\mathrm{SAM}$ upon isomerization of the switch from SP to MC. In fact, ring-closed SP isomer is neutral and has low molecular dipole moment ( $\mu \sim 4-6 \mathrm{D}$ ) compared to the ring-open MC isomer. The latter is the hybrid between a quinoidal and zwitterionic form and has higher molecular dipole moment $(\mu \sim 14-$ 18 D). ${ }^{33}$ The charges related to zwitterionic MC may quench the PL via enhanced non-radiative Auger recombination, in agreement with previous works..$^{11}$

To cast light onto the electrical properties of the $\mathrm{SP} / \mathrm{WSe}_{2}$ hybrid, it has been integrated as channel material into FET devices. The devices revealed an $n$-type behavior with electron mobility of $12.4 \mathrm{~cm}^{2} \mathrm{~V}^{-1} \mathrm{~s}^{-1}$, which is typical for back-gated $\mathrm{WSe}_{2}$ FETs with 9 atomic layers (Figure S12).${ }^{39}$ Interestingly, upon UV light irradiation to PSS condition, the device showed a major decrease of electron current with a modulation efficiency of $82 \%$ and the ultimate mobility dropped to $1.1 \mathrm{~cm}^{2} \mathrm{~V}^{-1} \mathrm{~s}^{-1}$. Upon heating, the current was restored almost to the initial value, in accordance with the reversible change in the absorption spectra and PL mapping. 
Inspired by our recent work on the dual functionalization of $\mathrm{WSe}_{2}$ with a ferroelectric copolymer in combination and a physisorbed photochromic diarylethenes layer to achieve ternary switchable field-effect transistors, ${ }^{40}$ in order to add an additional functionality to the asfabricated optically switchable SP/WSe $\mathrm{WET}_{2}$ FEvices, the upper surface of $\mathrm{WSe}_{2}$ flakes was dressed with a $580 \mathrm{~nm}$ thick layer of ferroelectric copolymer layer based on poly(vinylidene fluoride-co-trifluoroethylene) - P(VDF-TrFE) (Figure S13), followed by the deposition of a semi-transparent top-gate electrode (20 nm thick Au layer). Note that such thickness of P(VDFTrFE) is to guarantee the device's success rate during fabrication, as in agreement with previous reports. ${ }^{41,42}$ By applying a voltage to the upper $\mathrm{Au}$ electrode, a control over the polarization direction of the $\mathrm{P}(\mathrm{VDF}-\mathrm{TrFE})$ layer becomes possible (Figure S14). Figure 1b displays a schematic view of the SP/WSe $2 / \mathrm{P}(\mathrm{VDF}-\mathrm{TrFE})$ FET. Firstly, the device properties in top-gate configuration with $\mathrm{P}(\mathrm{VDF}-\mathrm{TrFE})$ serving as dielectric material were investigated (Figure S15a), revealing an electron transport-dominated ambipolar behavior. The corresponding leakage current is reported in Figure S15b, with two apparent current peaks near the coercive field of $\mathrm{P}(\mathrm{VDF}-\mathrm{TrFE})$, proving its good dielectric and ferroelectric characteristics.

The optical/heating switching capabilities of SP/WSe $2 / \mathrm{P}(\mathrm{VDF}-\mathrm{TrFE}) \mathrm{FET}$ were investigated in a back-gate device configuration (Figure $4 a-b)$. With its dielectric constant $\left(\varepsilon_{\mathrm{r}}=10\right)$ being higher than that of $\mathrm{SiO}_{2}\left(\varepsilon_{\mathrm{r}}=3.9\right), \mathrm{P}(\mathrm{VDF}-\mathrm{TrFE})$ can suppress the Coulomb scattering in $\mathrm{WSe}_{2}{ }^{2}$ As a consequence, an improved electron mobility $\left(18.2 \mathrm{~cm}^{2} \mathrm{~V}^{-1} \mathrm{~s}^{-1}\right)$ was observed in $\mathrm{SP} / \mathrm{WSe}_{2} / \mathrm{P}(\mathrm{VDF}-\mathrm{TrFE})$ FET compared to above $\mathrm{SP} / \mathrm{WSe} \mathrm{F}_{2}$ FET $\left(12.4 \mathrm{~cm}^{2} \mathrm{~V}^{-1} \mathrm{~s}^{-1}\right)$. In response to UV irradiation to PSS condition, the electron current notably decreased with a modulation efficiency of $84 \%$, along with a decrease of field-effect mobility up to $2.6 \mathrm{~cm}^{2} \mathrm{~V}^{-1} \mathrm{~s}^{-1}$. Figure $4 \mathrm{~b}$ depicts the reversible variation of the current during three cycles when different inputs were used to switch back the molecules to SP form: heating, vis irradiation and thermally at room temperature. By heating at $80{ }^{\circ} \mathrm{C}$ for $10 \mathrm{~min}$ or vis irradiation at $530 \mathrm{~nm}$ for $30 \mathrm{~min}$, the effects 
of the previous UV irradiation could be completely removed, and the electrical performance was restored to the original state. Conversely, by leaving the previously UV irradiated device overnight in dark at room temperature, only $63 \%$ of the initial current was restored. Importantly, such kinetics information from electrical characteristics matched the UV-vis absorption spectra results reported above.

We also explored the reversibility of device performance when $\mathrm{P}(\mathrm{VDF}-\mathrm{TrFE})$ exhibits upward or downward polarization directions (Figure 4c-e). When a $-80 \mathrm{~V}$ pulse was applied to the topgate electrode, dipoles inside $\mathrm{P}(\mathrm{VDF}-\mathrm{TrFE})$ were aligned along the external electric field in the upward direction with negatively charged C-F bond facing the channel, causing the pronounced depletion of electrons and accumulation of holes. The corresponding schematic representation of the polarization direction is given in Figure 4c. Therefore, compared to the pristine state (orange line), there was a dramatic decrease of electron current with an efficiency as high as 99\%, and a corresponding increase of hole current. The device exhibited more balanced ambipolar behavior, with hole current comparable to the electron current $\left(\mu_{\mathrm{h}+}=0.118 \mathrm{~cm}^{2} \mathrm{~V}^{-1} \mathrm{~s}^{-1}\right.$, $\left.\mu_{\mathrm{e}-=} 0.024 \mathrm{~cm}^{2} \mathrm{~V}^{-1} \mathrm{~s}^{-1}\right)$, compared to the initial $n$-type dominant transport. On the contrary, a +80 $\mathrm{V}$ pulse led to a reversed arrangement of dipole moments in the downward polarization direction. In this case, with positively charged C-H bonds facing the channel, the current state resumed to the original state. Such a reversible tuning could be repeated for at least 3 cycles without any attenuation upon the periodic $+80 \mathrm{~V}$ and $-80 \mathrm{~V}$ bias operation.

To prove the compatibility between the two aforementioned switching elements, we combined light and electric bias stimuli in a single switching cycle. As shown in Figure 4f-g, upon UV irradiation, the device current decreased in the same ratio as the results above (i.e., $84 \%$ ). Interestingly, after being exposed to a subsequent negative top-gate bias, the device showed a further electron current decrease, in combination with an increase of the hole current. Hence, 
the electrons in the channel were totally depleted and the device, in its final state, turned into p-type transport. Subsequent vis irradiation and positive bias led to the recovery of the original device performance. To further investigate our systems and prove the highest versatility in the use of the different independent inputs to modulate the device characteristics, the stimuli order was reversed to firstly polarize the $\mathrm{P}(\mathrm{VDF}-\mathrm{TrFE})$ into the upward direction, and then switching the SP molecules into MC (Figure 4h). This experiment displayed identical results to the previous one (Figure 4i), confirming that the two switching elements are able to work independently without mutual interference.

To better illustrate the current modulation in our SP/WSe $2 / \mathrm{P}(\mathrm{VDF}-\mathrm{TrFE})$ FET achieved by different stimuli, dynamic current variation was tracked when the top-gate bias and light irradiation were supplied separately as input signals. As shown in Figure S16, when a negative bias was applied, the device current greatly reduced, and remained constant at that state. Then, in response to the applied positive bias, a current spike appeared, followed by the recovery of the original performance. The reversible current modulation confirmed the polarization of $\mathrm{P}(\mathrm{VDF}-\mathrm{TrFE})$. Later, the UV irradiation triggered the photoisomerization of SP, thereby lowering the device current. Such current modulation cycle can be operated for at least 5 times, and a stepwise decay of current was clearly observed upon each cycle. These results further demonstrated that the $\mathrm{SP} / \mathrm{WSe}_{2} /(\mathrm{PVDF}-\mathrm{TrFE}) \mathrm{FET}$ device is able to orthogonally respond to both external electric field and light irradiation.

Upon confirmation of successful photoswitching of the SAM at the interface between $\mathrm{SiO}_{2}$ and active material, along with the consequent modulation in the electrical properties of our devices, we investigated the mechanism related to this phenomenon. We firstly hypothesized that electron transfer could occur between $\mathrm{MC}$ and $\mathrm{WSe}_{2}$. To analyze this possible physical process, we have evaluated the energy levels of $\mathrm{WSe}_{2}$ and SP/MC. The conduction band (CB) and 
valence band (VB) values of $\mathrm{WSe}_{2}$ were calculated by screened exchange functional method, ${ }^{43}$ and the highest occupied molecular orbital (HOMO) and lowest unoccupied molecular orbital (LUMO) energy levels of SP and MC were estimated by cyclic voltammetry (Figure S17). The energy level diagram is reported in Figure S18. Our devices based on few-layer $\mathrm{WSe}_{2}$ displayed electron-dominant transport behavior, so we focused our attention to the $\mathrm{CB}$ of $\mathrm{WSe}_{2}$ and the LUMO of SP/MC. Since the CB of $\mathrm{WSe}_{2}$ is lower than the LUMO of both SP and MC isomer, electrons in $\mathrm{WSe}_{2}$ can transfer to neither of these two isomers, hence the possibility for the MC molecules to act as electron trap sites can be excluded. On the other hand, when it comes to mono/bilayer $\mathrm{WSe}_{2}$ which possesses hole-transport behavior, its $\mathrm{VB}$ and the $\mathrm{HOMO}$ of SP/MC should be taken into consideration. In this case, the HOMO of MC is slightly higher than the $\mathrm{VB}$ of $\mathrm{WSe}_{2}$, allowing the MC isomer to act as a shallow trap for holes, which might result in a drop of hole current in the corresponding device. Electrical characterization on monolayer $\mathrm{WSe}_{2}$ was presented in Figure S19, which shows a reversible hole current modulation. Although this mechanism can partially explain the hole current modulation induced by SP SAM, it does not explain the above observed significant electron transport modulation, therefore we finally tested the ionic impurity scattering mechanism as previously reported for analogous systems. ${ }^{44}$ It is generally agreed that the charge carrier mobility/current of a semiconducting material is limited by the interfacial charged impurities, i.e. a lower current state is indicative of a higher density of charge scattering centers.$^{45}$ In our study, the SP isomer is neutral while the MC isomer is the hybrid between a quinoidal and zwitterionic form and has higher molecular dipole moment compared to SP. The high dipole moment MC isomers in the SAM may serve as scattering centers, inducing an attractive or repulsive force with the electrons flowing in the channel. Due to such Coulomb interactions, the ballistic acceleration of the flowing electrons in $\mathrm{WSe}_{2}$ by the electric field is disturbed, resulting in a change of trajectory and gradually scattering, the overall device current is thus being reduced. Such a 
model is similar to that of Coulomb scattering by ionic impurities in semiconducting materials..$^{46}$

To confirm such a hypothesis, we investigated the hysteresis behavior in our devices by varying the gate sweep rate and the overall sweep range, in bare $\mathrm{WSe}_{2} \mathrm{FET}$, and $\mathrm{SP} / \mathrm{WSe}_{2} \mathrm{FET}$ when the SP monolayer is either in the SP form or switched to MC in the UV PSS condition (Figure S20). The hysteresis in the transfer characteristics of 2DM-based FET devices has been attributed to several mechanisms, such as adsorption of $\mathrm{O}_{2}$ and $\mathrm{H}_{2} \mathrm{O}$ molecules, ${ }^{47,48}$ gate-oxide ${ }^{49}$ and oxide-TMD interface traps,$^{50}$ gate voltage stress ${ }^{51}$ and intrinsic defects in the material.$^{52}$ It is worth mentioning that in our system the only variable was the photochromic layer, as the experimental conditions and measurement environment were kept identical. A full gate sweep consists of both forward and backward sweep, i.e., from negative gate voltage $\left(\mathrm{V}_{\mathrm{bg}}\right)$ to positive $\mathrm{V}_{\mathrm{bg}}$ and then back to negative $\mathrm{V}_{\mathrm{bg}}$. A similar trend can be found for all the measured devices: $\mathrm{a}$ hysteresis with clockwise loop direction, the magnitude of which was tuned via both gate voltage sweep range and sweep rate. Figure 5a illustrates the comparison of the hysteresis for various sweep rates. In $\mathrm{WSe}_{2}$ FETs, the hysteresis was relatively small, with significant dependence on the sweep rate: it increased from $3 \mathrm{~V}$ at $3.2 \mathrm{~V} / \mathrm{s}$ to $22 \mathrm{~V}$ at $0.7 \mathrm{~V} / \mathrm{s}$. This is in accordance with the previous reports, where faster sweep rates inhibit the electron trapping during the measurements. ${ }^{53}$ Larger hysteresis was observed in $\mathrm{SP} / \mathrm{WSe}_{2}$ and $\mathrm{MC} / \mathrm{WSe}_{2}$, while the variation in magnitude with the sweep rate was minimal. Therefore, the main contribution to the hysteretic behavior can be ascribed to the SP/MC layer. Ionic impurity scattering is considered the main interaction between ionic adsorbates and the charge carriers in 2DMs. Moreover, it may alter the charge distribution at the interface between $\mathrm{SiO}_{2}$ and $2 \mathrm{DM}$ due to the field induced polarization effect, considered one of the major sources of hysteresis in FET devices. ${ }^{54,55}$ When comparing the hysteresis in $\mathrm{SP} / \mathrm{WSe}_{2}$ and $\mathrm{MC} / \mathrm{WSe}_{2}$, a more pronounced magnitude was observed in the latter. Such a result matches our assumption for which the 
zwitterionic MC isomer behaves as major scattering center. Note that for better comparison of their hysteresis magnitude and current evolution efficiency, transfer curves of $\mathrm{SP} / \mathrm{WSe} \mathrm{H}_{2}$ and $\mathrm{MC} / \mathrm{WSe}_{2}$ with the same scan rate have been plotted in Figure S21, both in logarithm and linear scale.

Besides sweep rate, the hysteresis variations were also investigated within different sweep ranges (Figure $\mathrm{S} 22$ ). For $\mathrm{WSe}_{2}$, the hysteresis was negligible in the sweep range of $30 \mathrm{~V}$, and its magnitude turned out to get larger as the range became wider. Such an observation indicates that hysteresis mainly originates from charge trapping/detrapping process at higher gate bias. ${ }^{52}$ When it comes to $\mathrm{SP} / \mathrm{WSe}_{2}$, the hysteresis variation became more prominent. These variations are plotted in Figure 5b, where molecules in MC form show a stronger sweep range dependent hysteresis variation compared to $\mathrm{SP} / \mathrm{WSe}_{2}$, thereby confirming that the $\mathrm{MC}$ isomer acts as ionic scattering center that plays a major role in the device performance.

\section{Conclusions}

In conclusion, we built quaternary-responsive $\mathrm{WSe}_{2}$ FETs by dressing asymmetrically its bottom and top surface with light-responsive SP molecules and ferroelectric co-polymer $\mathrm{P}(\mathrm{VDF}-\mathrm{TrFE})$, respectively. The output current of our SP/WSe $/ \mathrm{P}(\mathrm{VDF}-\mathrm{TrFE})$ FETs could be finely and independently modulated by external stimuli including heating, light irradiation and electric field. It is worth mentioning that the synergistic effect between the different stimuli enables a significant modulation of the charge carrier transport within the channel, switching from n-type to $p$-type with electrons being fully depleted. We also investigated the mechanism underlying the light induced transport modulation, highlighting how the zwitterionic MC isomer plays a major role as charge scattering center. Our design strategy of Janus modified structures via double-sided interfacial engineering can be extended to other 2DMs and to other 
stimuli which are introduced by the use of suitably designed (macro)molecular components, therefore offering a general guideline for the fabrication of multifunctional and multiresponsive FET devices. 
(a)

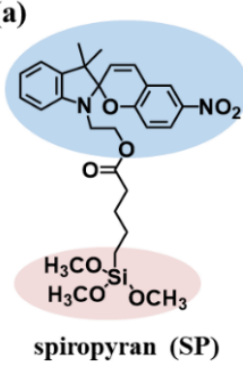

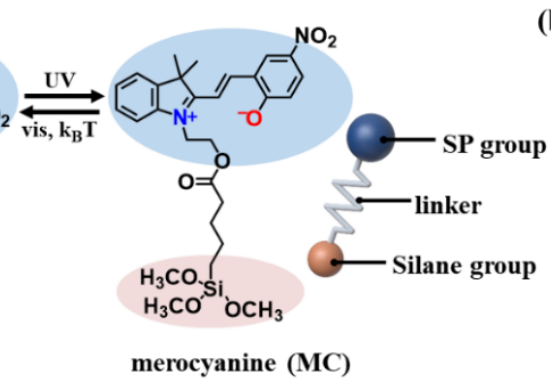

(b) $\circ: \mathrm{C} \bullet: \mathrm{H} \bullet: \mathrm{F}$

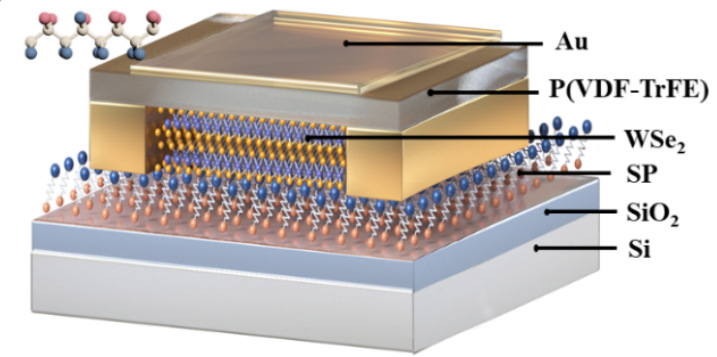

Figure 1: (a) Structure formulae of SP and MC and scheme of the SP - MC reversible isomerization obtained upon irradiation with $\mathrm{UV}(\lambda=365 \mathrm{~nm})$ and vis $(\lambda=530 \mathrm{~nm})$ light, respectively. (b) Schematic illustration of the Janus SP/WSe $2 / \mathrm{P}(\mathrm{VDF}-\mathrm{TrFE})$ FET device with double-sided decoration: the bottom surface of $\mathrm{WSe}_{2}$ is functionalized with SP SAM and the top surface is functionalized with $\mathrm{P}(\mathrm{VDF}-\mathrm{TrFE})$ layer. 
(a)
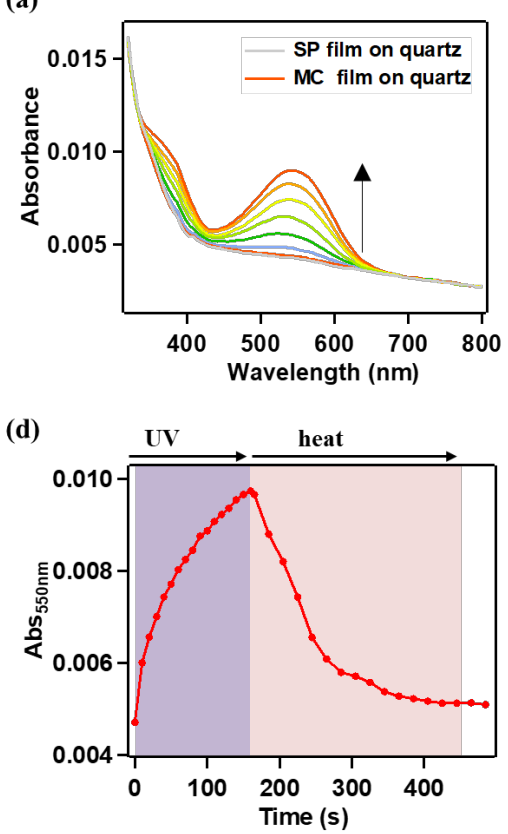

(b)

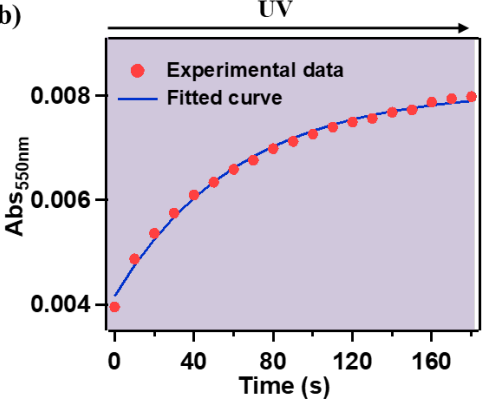

(e)

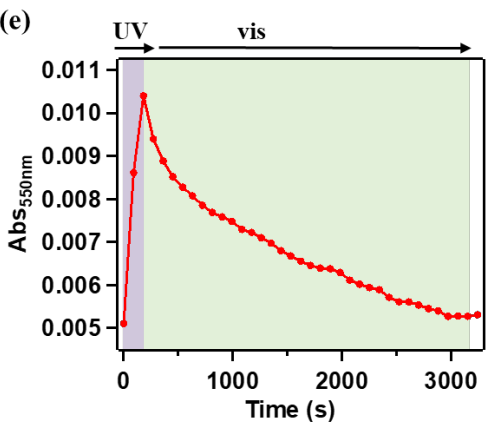

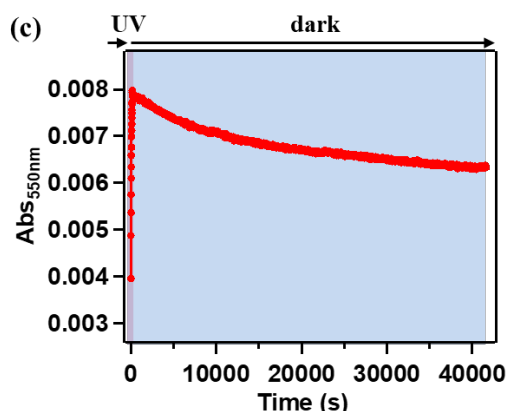

(f)

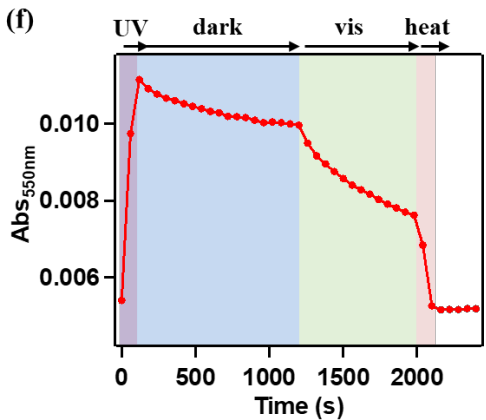

Figure 2: Optical characterization of the SP SAM photoisomerization on quartz substrate by UV-vis absorption spectroscopy. (a) Absorption spectral variation of SP SAM during the course of UV irradiation at $365 \mathrm{~nm}$ until reaching the photostationary state (PSS). (b-f) Kinetics of the SP SAM isomerization, monitored by plotting the absorbance at $550 \mathrm{~nm}$ under various conditions: (b) UV irradiation, (c) dark at room temperature, (d) dark upon heating at ca. 80 ${ }^{\circ} \mathrm{C}$, (e) vis irradiation at $530 \mathrm{~nm}$, (f) combination of dark, vis irradiation and heat. 
(a)

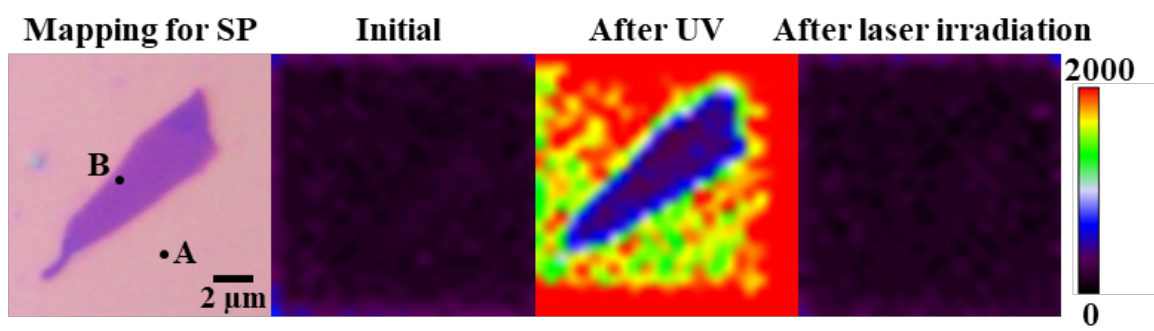

(b)
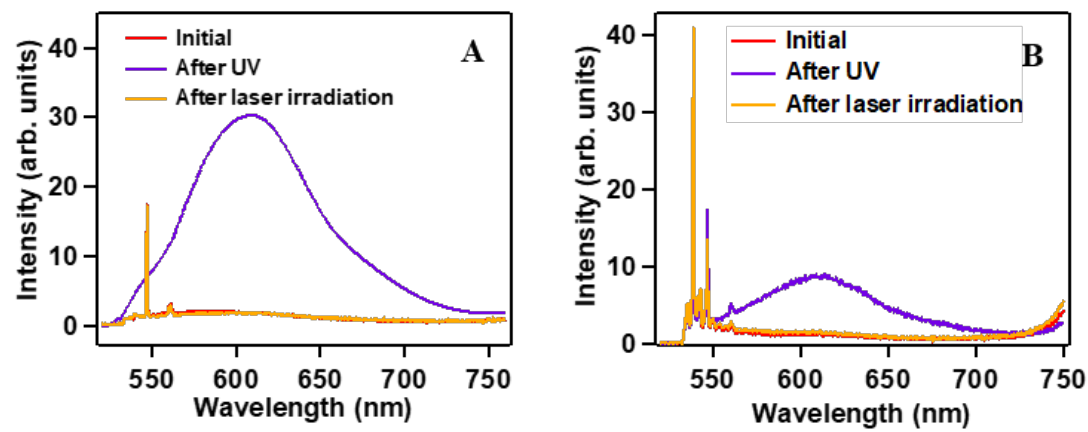

(c)

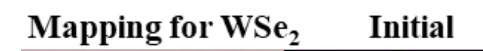

After UV After laser irradiation

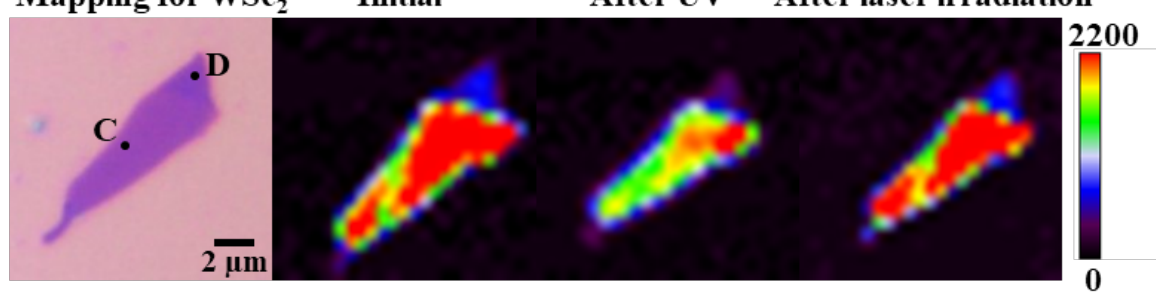

(d)
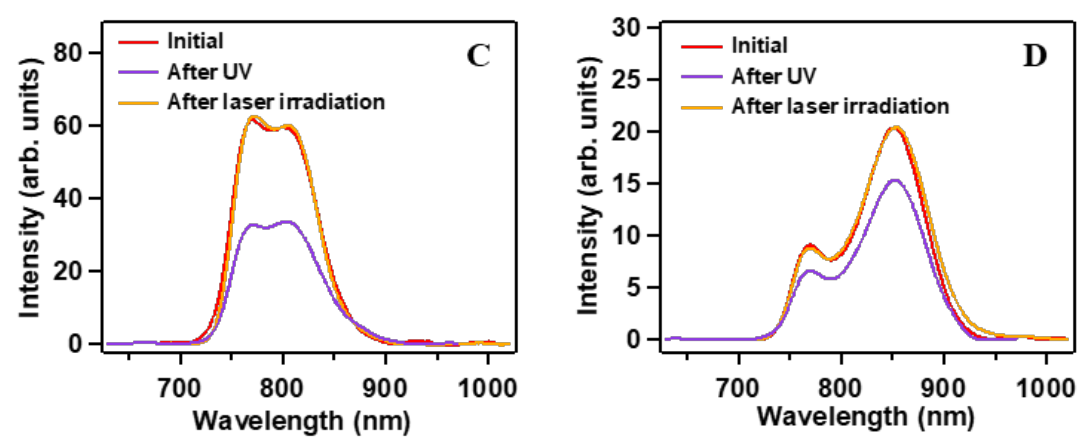

Figure 3: (a) Optical microscopy image of a SP/WSe ${ }_{2}$ flake (left) and its PL intensity mapping (right) recorded from $560 \mathrm{~nm}$ to $650 \mathrm{~nm}$ in initial, after UV irradiation, and after laser irradiation condition. (b) PL spectra evolution in the points A and B. (c) Same flake with PL intensity mapping recorded from $750 \mathrm{~nm}$ to $900 \mathrm{~nm}$ in initial, after UV irradiation, and after laser irradiation condition. (d) PL spectra evolution in the points C (bilayer) and D (few-layer). For all PL measurements, $\lambda_{\mathrm{exc}}=532 \mathrm{~nm}$. 
(a)

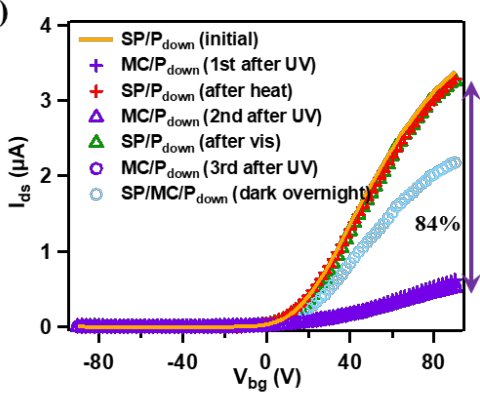

(d)

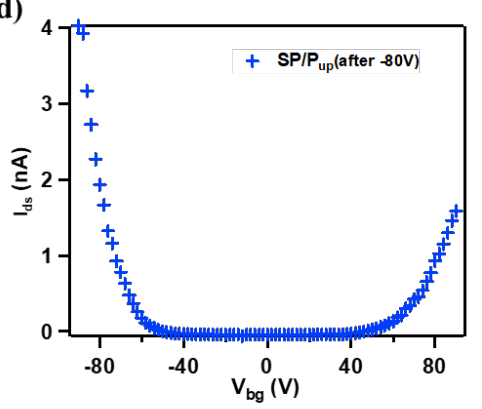

(g)

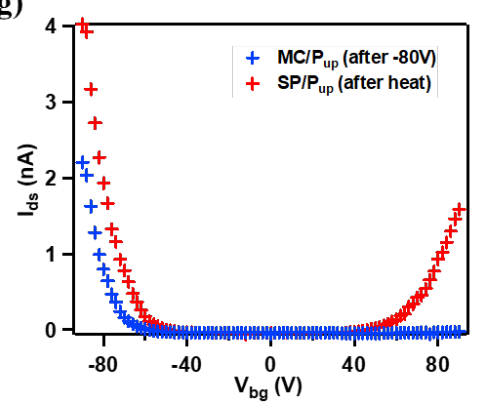

(b)

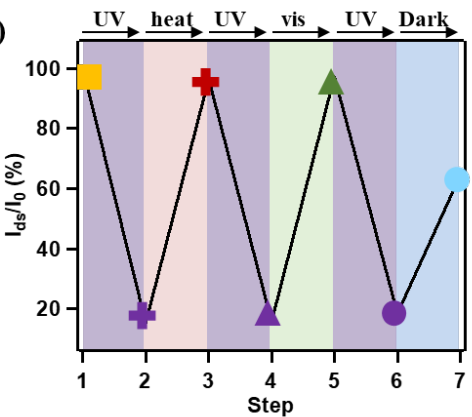

(e)

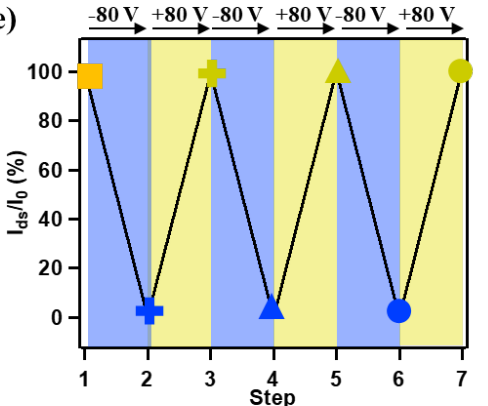

(h)

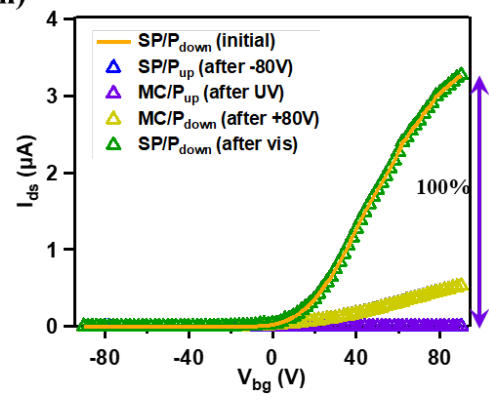

(c)

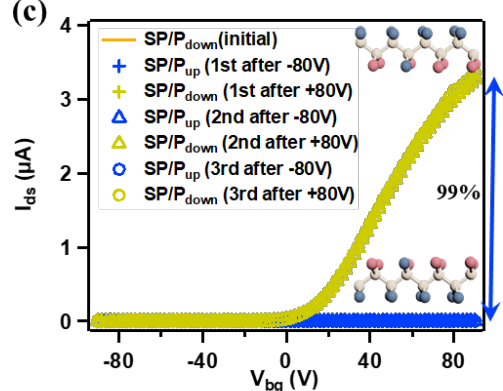

(f)

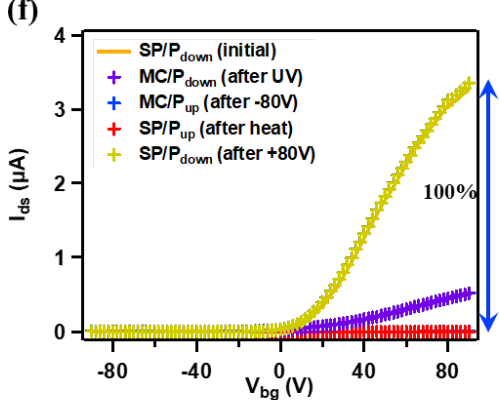

(i) $\stackrel{\mathrm{UV}}{\longrightarrow} \stackrel{80 \mathrm{~V}}{\longrightarrow} \stackrel{\text { hea }}{\longrightarrow}+80 \mathrm{~V}-80 \mathrm{VuV}+80 \mathrm{~V}$ vis

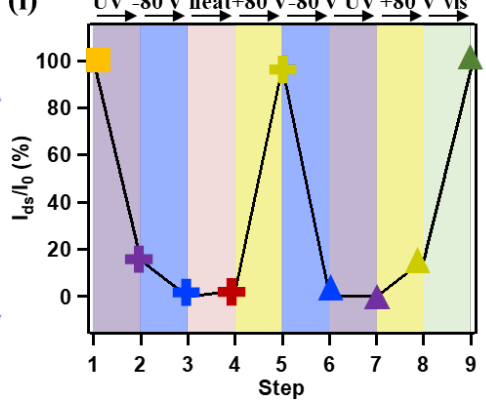

Figure 4: Electrical characterization of $\mathrm{SP} / \mathrm{WSe} / \mathrm{P}(\mathrm{VDF}-\mathrm{TrFE}) \mathrm{FET}$. (a) Optical/thermal switching by $\mathrm{UV}$, and heat/vis/dark treatment. (b) Current modulation efficiency at $\mathrm{V}_{\mathrm{bg}}=90 \mathrm{~V}$ during 3 switching cycles in (a). (c) Electrical switching by $\pm 80 \mathrm{~V}$ top-gate bias. (d) Transfer curve of SP/P up state in (c). (e) Current modulation efficiency at $\mathrm{V}_{\mathrm{bg}}=90 \mathrm{~V}$ during the 3 switching cycles in (c). (f) Orthogonal switching with the stimuli order of UV/-80 V/heat/+80 V. (g) Transfer curves of MC/P up state and $\mathrm{SP} / \mathrm{P}_{\text {up }}$ state in (f). (h) Orthogonal switching with the order of $-80 \mathrm{~V} / \mathrm{UV} /+80 \mathrm{~V} / \mathrm{vis}$. (i) Current modulation efficiency at $\mathrm{V}_{\mathrm{bg}}=90 \mathrm{~V}$ during the 2 switching cycles in (f) and (h). 

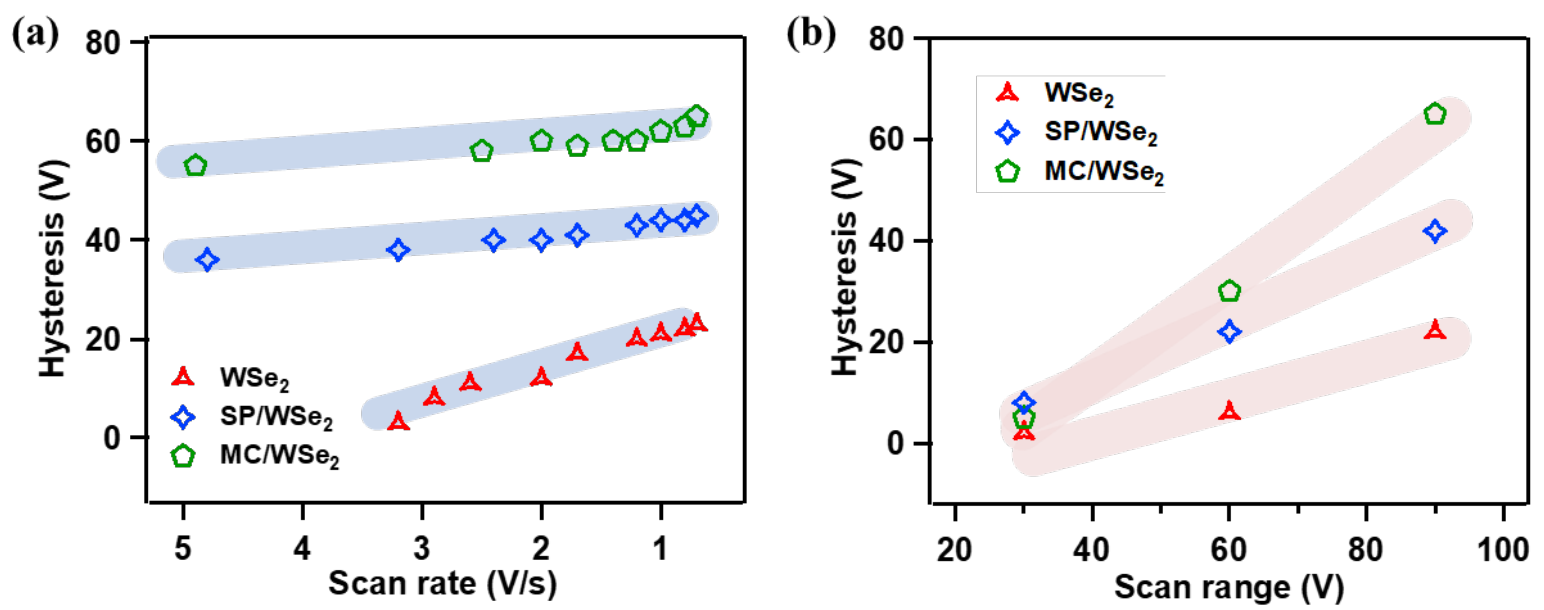

Figure 5. Hysteresis characterization. (a-b) Hysteresis comparison of FETs based on bare $\mathrm{WSe}_{2}, \mathrm{SP} / \mathrm{WSe}_{2}$ and $\mathrm{MC} / \mathrm{WSe}_{2}$ under (a) different scan rates, (b) different scan ranges. 


\section{Experimental methods}

Materials: $\mathrm{WSe}_{2}$ crystals were purchased from HQ graphene. The $\mathrm{P}(\mathrm{VDF}-\mathrm{TrFE})$ powder (with $70 \mathrm{~mol} \% \mathrm{VDF}$ ) was purchased from Solvay. $\mathrm{Si} / \mathrm{SiO}_{2} \mathrm{n}++$ substrates with $270 \mathrm{~nm}$ thick oxide were purchased from Fraunhofer Institute for Photonic Microsystems IPMS. The quartz substrates with $2 \mathrm{~mm}$ thickness were purchased from Plan Optik.

Synthesis of silane-terminated SP derivative. The synthetic strategy adopted for the preparation of the photochromic silane SP is reported in detail in the Supporting Information.

SP SAM formation. The formation of SP SAM via silylation of hydroxyl functions on $\mathrm{SiO}_{2}$ and quartz substrates with the photochromic silane SP is sketched in Figure S5. The substrates were initially cleaned in an ultrasonic bath in acetone and 2-propanol for $20 \mathrm{~min}$, respectively. Then they were activated by exposing to $\mathrm{UV} / \mathrm{O}_{3}$ for $20 \mathrm{~min}$ to maximize the number of reactive hydroxyl groups on the surface. Afterwards, the freshly $\mathrm{UV} / \mathrm{O}_{3}$ treated substrates were immediately immersed in a SP solution $(\mathrm{c}=3.6 \mathrm{mM})$ in toluene. The solution was heated at $60{ }^{\circ} \mathrm{C}$ for one hour and then left at room temperature overnight. Then, the substrates were thoroughly rinsed with toluene, and sonicated $5 \mathrm{~s}$ in hexane to remove the excess (unbound) SP. Finally, the substrates were baked at $60{ }^{\circ} \mathrm{C}$ for one hour in order to polymerize the SP monolayer.

UV-vis absorption spectra. UV-vis absorption spectra and isomerization kinetics of the SP solution/SAM on quartz substrates were recorded at room temperature with a Jasco V650 spectrophotometer. The photoswitching experiments were performed with optical fibercoupled LEDs (from ThorLabs) with wavelength of $365 \mathrm{~nm}\left(1.2 \mathrm{~mW} / \mathrm{cm}^{2}\right)$ or $\lambda=530 \mathrm{~nm}(4$ $\mathrm{mW} / \mathrm{cm}^{2}$ ). The switching by heating was performed using a heat gun set at $c a .80^{\circ} \mathrm{C}$. The PSS state is reached within 3 min of UV illumination. For SP solution, UV illumination intensities have been varied from 0.2 to $1 \mathrm{~mW} / \mathrm{cm}^{2}$, to examine the different saturation time of SP to MC 
transition. For SP SAM on quartz substrate, intensities have been varied from 0.4 to 1.9 $\mathrm{mW} / \mathrm{cm}^{2}$. For the photoisomerization kinetics, in situ irradiation was performed within the spectrophotometer chamber, by shining UV/vis light on the quartz substrate using a custommade setup. UV/vis irradiation was performed for fixed intervals, and $\mathrm{Abs}_{550 \mathrm{~nm}}$ was recorded immediately after switching off the light.

Water contact angle. The measurement was performed with a Krüss DSA 100, by deposition of a $2 \mu \mathrm{L}$ drop of Milli-Q water on unmodified $\mathrm{Si} / \mathrm{SiO}_{2}$, pristine SP SAMs, and UV/heat-treated SP SAMs on $\mathrm{Si} / \mathrm{SiO}_{2}$ surfaces. The UV illumination was performed with optical fiber-coupled LEDs (ThorLabs) with wavelength of $365 \mathrm{~nm}\left(1.2 \mathrm{~mW} / \mathrm{cm}^{2}\right)$ for $3 \mathrm{~min}$. The heating was performed using a heat gun set at $c a .80{ }^{\circ} \mathrm{C}$ for $4 \mathrm{~min}$. Note that each measurement was repeated for 3 times and the average value was calculated.

AFM Imaging. The measurements were carried out using a Bruker Dimension Icon microscope in ambient conditions, operating in tapping mode and using TESPA-V2 tips with spring constant $\mathrm{k}=42 \mathrm{~N} / \mathrm{m}$.

PL mapping. The PL measurements were performed with a Renishaw InVia Raman microscope equipped with a $532 \mathrm{~nm}$ laser line. Each mapping was carried out with $0.5 \mathrm{~mm}$ steps in an area of $15 \times 15 \mathrm{~mm}^{2}$. The spectra were recorded with $0.1 \mathrm{~s}$ of integration time and 1 accumulation, under laser power of $0.03 \mathrm{~mW}$. To switch the molecules from MC to SP form, the same area was scanned using the same operating conditions and parameters but with a higher laser power $(4.92 \mathrm{~mW})$.

Device Fabrication and Characterization. After functionalizing the substrates with SP SAM, the mechanically exfoliated few-layer $\mathrm{WSe}_{2}$ flakes were transferred onto the SP-decorated substrates. The source and drain electrodes were defined by Microtech laser writer equipped with a $405 \mathrm{~nm}$ laser. The AZ1505 photoresist and AZ326 MIF developer from MicroChemicals 
were used during the process. Then $60 \mathrm{~nm}$ Au was deposited onto the substrate followed by lift-off in warm acetone $\left(\mathrm{ca} .55^{\circ} \mathrm{C}\right)$. The P(VDF-TrFE) solution (10\% wt in butanol) was spincoated on top of the SP/WSe ${ }_{2}$ device $(1000 \mathrm{rpm}, 60 \mathrm{~s})$, followed by annealing at $140{ }^{\circ} \mathrm{C}$ for 2 hours on a hotplate in the nitrogen-filled glovebox to enhance its crystallinity. As verified by the transfer evolution of the SP/WSe ${ }_{2}$ device before and after curing step (Figure S23), the SP modulation will not be deteriorated during such high-temperature annealing. Finally, a semitransparent top-gate electrode $(20 \mathrm{~nm} \mathrm{Au})$ was evaporated on top of the P(VDF-TrFE) layer.

The electrical characterization was performed in the glovebox with a probe station connected to a Keithley 2636 sourcemeter. Light illumination for switching the SP was performed using an optical fiber-coupled monochromator (UV light at $\lambda=365 \mathrm{~nm}, \mathrm{P}_{\mathrm{d}}=3.2 \mathrm{~mW} / \mathrm{cm}^{2}$ for $6 \mathrm{~min}$ to reach PSS or vis light at $\lambda=530 \mathrm{~nm}, \mathrm{P}_{\mathrm{d}}=11.8 \mathrm{~mW} / \mathrm{cm}^{2}$ for $30 \mathrm{~min}$ to recover to SP). Heating for switching was performed using the hotplate at $80{ }^{\circ} \mathrm{C}$ for $10 \mathrm{~min}$.

The charge carrier mobility is calculated in the saturation regime from the transfer curve by using the expression: ${ }^{56}$

$\mu=\frac{\mathrm{dI}_{\mathrm{ds}}}{\mathrm{dV}_{\mathrm{g}}} \times \frac{\mathrm{L}}{\mathrm{W}} \times \frac{1}{\mathrm{~V}_{\mathrm{ds}}} \times \frac{d}{\varepsilon_{0} \varepsilon_{r}}$

Where $\mathrm{L}$ is the channel length, $\mathrm{W}$ is the channel width, and $\varepsilon_{\mathrm{r}}$ is the dielectric constant, $\varepsilon_{\mathrm{r}}=$ 3.9 for $\mathrm{SiO}_{2}$ and $\varepsilon_{\mathrm{r}}=10$ for $\mathrm{P}(\mathrm{VDF}-\mathrm{TrFE})$.

Thickness determination. The thickness of the $\mathrm{P}(\mathrm{VDF}-\mathrm{TrFE})$ layer was measured by a profilometer. The $\mathrm{P}(\mathrm{VDF}-\mathrm{TrFE})$ layer was spin-coated to a clean $\mathrm{Si} / \mathrm{SiO}_{2}$ substrate, followed by 2 hours annealing at $140{ }^{\circ} \mathrm{C}$ in the glovebox. Note that the layer preparation recipe kept the same as in the $\mathrm{SP} / \mathrm{WSe}_{2} / \mathrm{P}(\mathrm{VDF}-\mathrm{TrFE}) \mathrm{FET}$ device fabrication process. Then a step was created by scratching the layer penetrating down to the substrate. The thickness was finally determined by measuring the depth between the layer and the clean substrate, which was $c a .580 \mathrm{~nm}$ (Figure S13). 
Cyclic voltammetry (CV). The measurements were performed with a three-electrode configuration cell, with platinum working electrode, platinum wire as counter electrode, and saturated Calomel electrode as reference electrode. Ferrocene/ferrocenium redox couple was employed as internal standard. The SP was dissolved in acetonitrile at a concentration of $10^{-3}$ M, and the solution was degassed by bubbling nitrogen for $20 \mathrm{~min}$. The scan rate during the CV experiments was kept at $100 \mathrm{mV} / \mathrm{s}$.

\section{ASSOCIATED CONTENT}

Supporting Information. The Supporting Information is available free of charge on the ACS Publications website at DOI:

Figures S1-S23, Table S1-S3, and references. Synthesis of silane-terminated SP derivative. SP $\mathrm{SAM}$ formation procedure. Fitting of $\mathrm{MC} \rightarrow \mathrm{SP} \mathrm{UV}$-vis absorption spectra. Dependence of saturation time for SP to MC transition on the UV illumination intensity. Water contact angle of unmodified $\mathrm{Si} / \mathrm{SiO}_{2}$ substrate, $\mathrm{SP}$ treated $\mathrm{Si} / \mathrm{SiO}_{2}$ substrate as initial and after $\mathrm{UV} /$ heat treatment. AFM imaging of $\mathrm{Si} / \mathrm{SiO}_{2}$ substrate and $\mathrm{SP}$ treated $\mathrm{Si} / \mathrm{SiO}_{2}$ substrate. PL mapping of $\mathrm{SP} / \mathrm{WSe}_{2}$. Characterization of SP/WSe $\mathrm{FET}_{2}$. Thickness measurement of the P(VDF-TrFE) layer. Optical microscopy images captured at four stages during the $\mathrm{SP} / \mathrm{WSe} \mathrm{S}_{2} / \mathrm{P}(\mathrm{VDF}-\mathrm{TrFE})$ FET fabrication process. Characterization of $\mathrm{SP} / \mathrm{WSe}_{2} / \mathrm{P}(\mathrm{VDF}-\mathrm{TrFE})$ FET in top-gate configuration. Characterization of $\mathrm{SP} / \mathrm{WSe}_{2} / \mathrm{P}(\mathrm{VDF}-\mathrm{TrFE}) \mathrm{FET}$ in back-gate configuration. Energy level alignment between $\mathrm{SP} / \mathrm{MC}$ and $\mathrm{WSe}_{2}$. Hole transport modulation in $\mathrm{SP} / \mathrm{WSe} \mathrm{FET}_{2}$ based on monolayer flake. Hysteresis characterization of FETs based on bare $\mathrm{WSe}_{2}, \mathrm{SP} / \mathrm{WSe}_{2}$ and $\mathrm{MC} / \mathrm{WSe}_{2}$ under different scan rates and scan ranges. Comparison of $\mathrm{SP} / \mathrm{WSe}_{2}$ device performance before and after the curing step. 


\section{AUTHOR INFORMATION}

\section{Corresponding Author}

*E-mail: samori@unistra.fr

\section{ORCID}

Paolo Samorì: 0000-0001-6256-8281

Stefano Ippolito: 0000-0002-6906-3961

Agostino Galanti: 0000-0003-2758-9508

Zhaoyang Liu: 0000-0001-7972-3452

\section{Author Contributions}

H.Q. and P.S. conceived the experiments and designed the study. A.G. designed and synthesized the spiropyran derivative. H.Q. performed the optical characterization, PL measurement, device fabrication and characterization. S.I. carried out the AFM study and contributed to the interpretation of electrical characterizations. Z.L. performed the CV measurement. All authors discussed the results and contributed to the interpretation of data. H.Q. and P.S. co-wrote the paper with input from all co-authors.

\section{ACKNOWLEDGMENTS}

We acknowledge funding from the European Commission through the ERC project SUPRA2DMAT (GA-833707) and the Graphene Flagship Core 3 project (GA- 881603), the Agence Nationale de la Recherche through the Labex projects CSC (ANR-10-LABX-0026 CSC) and NIE (ANR-11-LABX-0058 NIE) within the Investissement d'Avenir program (ANR-10-120 IDEX-0002-02), the International Center for Frontier Research in Chemistry (icFRC), the Institut Universitaire de France (IUF) as well as the Chinese Scholarship Council. 


\section{REFERENCES}

1. Novoselov, K. S.; Fal'ko, V. I.; Colombo, L.; Gellert, P. R.; Schwab, M. G.; Kim, K., A Roadmap for Graphene. Nature 2012, 490, 192-200.

2. Radisavljevic, B.; Radenovic, A.; Brivio, J.; Giacometti, V.; Kis, A., Single-Layer $\mathrm{MoS}_{2}$ Transistors. Nat. Nanotechnol. 2011, 6, 147.

3. Wang, Q. H.; Kalantar-Zadeh, K.; Kis, A.; Coleman, J. N.; Strano, M. S., Electronics and Optoelectronics of Two-Dimensional Transition Metal Dichalcogenides. Nat. Nanotechnol. 2012, 7, 699.

4. Wang, H.; Yuan, H.; Hong, S. S.; Li, Y.; Cui, Y., Physical and Chemical Tuning of Two-Dimensional Transition Metal Dichalcogenides. Chem. Soc. Rev. 2015, 44, 2664-2680.

5. Cai, Y.; Zhou, H.; Zhang, G.; Zhang, Y.-W., Modulating Carrier Density and Transport Properties of $\mathrm{MoS}_{2}$ by Organic Molecular Doping and Defect Engineering. Chem.Mater. 2016, $28,8611-8621$.

6. Li, Y.; Xu, C.-Y.; Hu, P.; Zhen, L., Carrier Control of $\mathrm{MoS}_{2}$ Nanoflakes by Functional Self-Assembled Monolayers. ACS Nano 2013, 7, 7795-7804.

7. Gobbi, M.; Orgiu, E.; Samorì, P., When 2D Materials Meet Molecules: Opportunities and Challenges of Hybrid Organic/Inorganic van der Waals Heterostructures. Adv. Mater. 2018, 30, 1706103.

8. Xu, C.; Zhang, J.; Xu, W.; Tian, H., Multifunctional Organic Field Effect Transistors Constructed with Photochromic Molecules. Mater. Chem. Front. 2021, 5, 1060-1075.

9. Leydecker, T.; Herder, M.; Pavlica, E.; Bratina, G.; Hecht, S.; Orgiu, E.; Samorì, P., Flexible Non-Volatile Optical Memory Thin-Film Transistor Device with over 256 Distinct Levels Based on an Organic Bicomponent Blend. Nat. Nanotechnol. 2016, 11, 769-775.

10. Lee, Y. T.; Kwon, H.; Kim, J. S.; Kim, H.-H.; Lee, Y. J.; Lim, J. A.; Song, Y.-W.; Yi, Y.; Choi, W.-K.; Hwang, D. K.; Im, S., Nonvolatile Ferroelectric Memory Circuit Using Black 
Phosphorus Nanosheet-Based Field-Effect Transistors with P(VDF-TrFE) Polymer. ACS Nano 2015, 9, 10394-10401.

11. Balcı Leinen, M.; Klein, P.; Sebastian, F. L.; Zorn, N. F.; Adamczyk, S.; Allard, S.; Scherf, U.; Zaumseil, J., Spiropyran-Functionalized Polymer-Carbon Nanotube Hybrids for Dynamic Optical Memory Devices and UV Sensors. Adv. Electron. Mater. 2020, 6, 2000717. 12. Sun,H.; Li, J.-Y.; Han, F.-F.; Zhang, R.; Zhao, Y.; Miao, B.-X.; Ni, Z.-H., Reversible Photochromic Tetraphenylethene-Based Schiff Base: Design, Synthesis, Crystal Structure and Applications as Visible Light Driven Rewritable Paper and UV Sensor. Dyes Pigm. 2019, 167, $143-150$.

13. Dai, S.; Wu, X.; Liu, D.; Chu, Y.; Wang, K.; Yang, B.; Huang, J., Light-Stimulated Synaptic Devices Utilizing Interfacial Effect of Organic Field-Effect Transistors. ACS Appl. Mater. Interfaces 2018, 10, 21472-21480.

14. Yu, Y.; Ma, Q.; Ling, H.; Li, W.; Ju, R.; Bian, L.; Shi, N.; Qian, Y.; Yi, M.; Xie, L.; Huang, W., Small-Molecule-Based Organic Field-Effect Transistor for Nonvolatile Memory and Artificial Synapse. Adv. Funct. Mater. 2019, 29, 1904602.

15. Brieke, C.; Rohrbach, F.; Gottschalk, A.; Mayer, G.; Heckel, A., Light - Controlled Tools. Angew. Chem. Int. Ed. 2012, 51, 8446-8476.

16. Zhao, Y.; Ippolito, S.; Samorì, P., Functionalization of 2D Materials with Photosensitive Molecules: From Light-Responsive Hybrid Systems to Multifunctional Devices. Adv. Opt. Mater. 2019, 7, 1900286.

17. Qiu, H.; Zhao, Y.; Liu, Z.; Herder, M.; Hecht, S.; Samorì, P., Modulating the Charge Transport in 2D Semiconductors via Energy-Level Phototuning. Adv. Mater. 2019, 31, 1903402.

18. Qiu, H.; Liu, Z.; Yao, Y.; Herder, M.; Hecht, S.; Samorì, P., Simultaneous Optical Tuning of Hole and Electron Transport in Ambipolar $\mathrm{WSe}_{2}$ Interfaced with a Bicomponent 
Photochromic Layer: From High-Mobility Transistors to Flexible Multilevel Memories. Adv. Mater. 2020, 32, 1907903.

19. Zhao, Y.; Bertolazzi, S.; Samorì, P., A Universal Approach toward Light-Responsive Two-Dimensional Electronics: Chemically Tailored Hybrid van der Waals Heterostructures. ACS Nano 2019, 13, 4814-4825.

20. Kim, M.; Safron, N. S.; Huang, C.; Arnold, M. S.; Gopalan, P., Light-Driven Reversible Modulation of Doping in Graphene. Nano Lett. 2012, 12, 182-187.

21. Margapoti, E.; Strobel, P.; Asmar, M. M.; Seifert, M.; Li, J.; Sachsenhauser, M.; Ceylan, Ö.; Palma, C.-A.; Barth, J. V.; Garrido, J. A.; Cattani-Scholz, A.; Ulloa, S. E.; Finley, J. J., Emergence of Photoswitchable States in a Graphene-Azobenzene-Au Platform. Nano Lett. 2014, 14, 6823-6827.

22. Margapoti, E.; Li, J.; Ceylan, Ö.; Seifert, M.; Nisic, F.; Anh, T. L.; Meggendorfer, F.; Dragonetti, C.; Palma, C.-A.; Barth, J. V.; Finley, J. J., A 2D Semiconductor-Self-Assembled Monolayer Photoswitchable Diode. Adv. Mater. 2015, 27, 1426-1431.

23. Gobbi, M.; Bonacchi, S.; Lian, J. X.; Vercouter, A.; Bertolazzi, S.; Zyska, B.; Timpel, M.; Tatti, R.; Olivier, Y.; Hecht, S.; Nardi, M. V.; Beljonne, D.; Orgiu, E.; Samorì, P., Collective Molecular Switching in Hybrid Superlattices for Light-Modulated TwoDimensional Electronics. Nat. Commun. 2018, 9, 2661.

24. Gobbi, M.; Galanti, A.; Stoeckel, M.-A.; Zyska, B.; Bonacchi, S.; Hecht, S.; Samorì, P., Graphene Transistors for Real-Time Monitoring Molecular Self-Assembly Dynamics. Nat. Commun. 2020, 11, 4731 .

25. Jang, A.-R.; Jeon, E. K.; Kang, D.; Kim, G.; Kim, B.-S.; Kang, D. J.; Shin, H. S., Reversibly Light-Modulated Dirac Point of Graphene Functionalized with Spiropyran. ACS Nano 2012, 6, 9207-9213. 
26. JoonáKim, B.; KyungáJeon, E.; HoáCho, J., Optical Switching of the Dirac Point in Graphene Multilayer Field-Effect Transistors Functionalized with Spiropyran. Chem. Commun. 2012, 48, 10978-10980.

27. Zhou, C.; Chai, Y., Ferroelectric-Gated Two-Dimensional-Material-Based Electron Devices. Adv. Electron. Mater. 2017, 3, 1600400.

28. Chen, X.; Han, X.; Shen, Q.-D., PVDF-Based Ferroelectric Polymers in Modern Flexible Electronics. Adv. Electron. Mater. 2017, 3, 1600460.

29. Lee, Y. T.; Hwang, D. K.; Im, S., High-Performance a $\mathrm{MoS}_{2}$ Nanosheet-Based Nonvolatile Memory Transistor with a Ferroelectric Polymer and Graphene Source-Drain Electrode. J. Korean Phys. Soc. 2015, 67, 1499-1503.

30. Wang, X.; Wang, P.; Wang, J.; Hu, W.; Zhou, X.; Guo, N.; Huang, H.; Sun, S.; Shen, H.; Lin, T.; Tang, M.; Liao, L.; Jiang, A.; Sun, J.; Meng, X.; Chen, X.; Lu, W.; Chu, J., Ultrasensitive and Broadband $\mathrm{MoS}_{2}$ Photodetector Driven by Ferroelectrics. Adv. Mater. 2015, $27,6575-6581$.

31. Li, D.; Wang, X.; Chen, Y.; Zhu, S.; Gong, F.; Wu, G.; Meng, C.; Liu, L.; Wang, L.; Lin, T.; Sun, S.; Shen, H.; Wang, X.; Hu, W.; Wang, J.; Sun, J.; Meng, X.; Chu, J., The Ambipolar Evolution of a High-Performance $\mathrm{WSe}_{2}$ Transistor Assisted by a Ferroelectric Polymer. Nanotechnology 2018, 29, 105202.

32. Stoeckel, M.-A.; Gobbi, M.; Leydecker, T.; Wang, Y.; Eredia, M.; Bonacchi, S.; Verucchi, R.; Timpel, M.; Nardi, M. V.; Orgiu, E.; Samorì, P., Boosting and Balancing Electron and Hole Mobility in Single- and Bilayer $\mathrm{WSe}_{2}$ Devices via Tailored Molecular Functionalization. ACS Nano 2019, 13, 11613-11622.

33. Klajn, R., Spiropyran-Based Dynamic Materials. Chem. Soc. Rev. 2014, 43, 148-184. 
34. Gómez, I.; Reguero, M.; Robb, M. A., Efficient Photochemical Merocyanine-toSpiropyran Ring Closure Mechanism through an Extended Conical Intersection Seam. A Model CASSCF/CASPT2 Study. J. Phys. Chem. A 2006, 110, 3986-3991.

35. Kortekaas, L.; Browne, W. R., The Evolution of Spiropyran: Fundamentals and Progress of an Extraordinarily Versatile Photochrome. Chem. Soc. Rev. 2019, 48, 3406-3424. 36. Lee, B.; Chen, Y.; Duerr, F.; Mastrogiovanni, D.; Garfunkel, E.; Andrei, E. Y.; Podzorov, V., Modification of Electronic Properties of Graphene with Self-Assembled Monolayers. Nano Lett. 2010, 10, 2427-2432.

37. Ivashenko, O.; van Herpt, J. T.; Feringa, B. L.; Rudolf, P.; Browne, W. R., UV/Vis and Nir Light-Responsive Spiropyran Self-Assembled Monolayers. Langmuir 2013, 29, 4290-4297.

38. Xu, K.; Wang, Z.; Du, X.; Safdar, M.; Jiang, C.; He, J., Atomic-Layer Triangular WSe 2 Sheets: Synthesis and Layer-Dependent Photoluminescence Property. Nanotechnology 2013, 24,465705 .

39. Pudasaini, P. R.; Oyedele, A.; Zhang, C.; Stanford, M. G.; Cross, N.; Wong, A. T.; Hoffman, A. N.; Xiao, K.; Duscher, G.; Mandrus, D. G.; Ward, T. Z.; Rack, P. D., HighPerformance Multilayer $\mathrm{WSe}_{2}$ Field-Effect Transistors with Carrier Type Control. Nano Res. 2018, $11,722-730$.

40. Qiu, H.; Liu, Z.; Herder, M.; Hecht,S.; Samorì, P., Ternary-Responsive Field-Effect Transistors and Multilevel Memories Based on Asymmetrically Functionalized Janus FewLayer $\mathrm{WSe}_{2}$. Adv. Funct. Mater. 2021, in press.

41. Zheng, Y.; Ni, G.-X.; Toh, C.-T.; Zeng, M.-G.; Chen, S.-T.; Yao, K.; Özyilmaz, B., Gate-Controlled Nonvolatile Graphene-Ferroelectric Memory. 2009, 94, 163505. 
42. Xiong, L.; Chen, Y.; Yu, J.; Xiong, W.; Zhang, X.; Zheng, Y., Stretchable Ferroelectric Field-Effect-Transistor with Multi-Level Storage Capacity and Photo-Modulated Resistance. 2019, $115,153107$.

43. Guo, Y.; Robertson, J., Band Engineering in Transition Metal Dichalcogenides: Stacked versus Lateral Heterostructures. Appl. Phys. Lett. 2016, 108, 233104.

44. Ishiguro, Y.; Hayakawa, R.; Chikyow, T.; Wakayama, Y., Optical Switching of Carrier Transport in Polymeric Transistors with Photochromic Spiropyran Molecules. J. Mater. Chem. C 2013, 1, 3012-3016.

45. Ando, T., Screening Effect and Impurity Scattering in Monolayer Graphene. J. Phys. Soc.Jpn. 2006, 75, 074716-074716.

46. Chattopadhyay, D.; Queisser, H. J., Electron Scattering by Ionized Impurities in Semiconductors. Rev. Mod. Phys 1981, 53, 745-768.

47. Late, D. J.; Liu, B.; Matte, H. S. S. R.; Dravid, V. P.; Rao, C. N. R., Hysteresis in Single-Layer $\mathrm{MoS}_{2}$ Field Effect Transistors. ACS Nano 2012, 6, 5635-5641.

48. Kaushik, N.; Mackenzie, D. M. A.; Thakar, K.; Goyal, N.; Mukherjee, B.; Boggild, P.; Petersen, D. H.; Lodha, S., Reversible Hysteresis Inversion in $\mathrm{MoS}_{2}$ Field Effect Transistors. NPJ 2D Mater. Appl. 2017, 1, 34.

49. Park, Y.; Baac, H. W.; Heo, J.; Yoo, G., Thermally Activated Trap Charges Responsible for Hysteresis in Multilayer $\mathrm{MoS}_{2}$ Field-Effect Transistors. Appl. Phys. Lett. 2016, 108, 083102.

50. Guo, Y.; Wei, X.; Shu, J.; Liu, B.; Yin, J.; Guan, C.; Han, Y.; Gao, S.; Chen, Q., Charge Trapping at the $\mathrm{MoS}_{2}-\mathrm{SiO}_{2}$ Interface and Its Effects on the Characteristics of $\mathrm{MoS}_{2}$ MetalOxide-Semiconductor Field Effect Transistors. Appl. Phys. Lett. 2015, 106, 103109. 
51. Cho, K.; Park, W.; Park, J.; Jeong, H.; Jang, J.; Kim, T.-Y.; Hong, W.-K.; Hong, S.; Lee, T., Electric Stress-Induced Threshold Voltage Instability of Multilayer $\mathrm{MoS}_{2}$ Field Effect Transistors. ACS Nano 2013, 7, 7751-7758.

52. Shu, J.; Wu, G.; Guo, Y.; Liu, B.; Wei, X.; Chen, Q., The Intrinsic Origin of Hysteresis in $\mathrm{MoS}_{2}$ Field Effect Transistors. Nanoscale 2016, 8, 3049-3056.

53. Park, J.-M.; Lee, D.; Shim, J.; Jeon, T.; Eom, K.; Park, B.-G.; Lee, J.-H., Pulsed I-V Measurement Method to Obtain Hysteresis-Free Characteristics of Graphene FETs. Semicond. Sci. Technol. 2014, 29, 095006.

54. Chen, S.-H.; Nguyen, Y.; Chen, T.-W.; Yen, Z.-L.; Hofmann, M.; Hsieh, Y.-P., Neutral Scatterers Dominate Carrier Transport in CVD Graphene with Ionic Impurities. Carbon 2020, $165,163-168$.

55. Egginger, M.; Irimia-Vladu, M.; Schwödiauer, R.; Tanda, A.; Frischauf, I.; Bauer, S.; Sariciftci, N. S., Mobile Ionic Impurities in Poly(Vinyl Alcohol) Gate Dielectric: Possible Source of the Hysteresis in Organic Field-Effect Transistors. Adv. Mater. 2008, 20, 1018-1022. 56. Liu, H.; Neal, A. T.; Ye, P. D., Channel Length Scaling of $\mathrm{MoS}_{2}$ MOSFETS. ACS Nano 2012, $6,8563-8569$. 